\title{
CD5 positive breast carcinoma in a patient with untreated chronic lymphocytic leukaemia: molecular studies of chromosome 13q
}

\author{
S Shousha, Christine Costello, Y A Luqmani, H D Sinnett
}

\begin{abstract}
A 67 year old woman presented with a right breast lump which proved to be a grade 2 invasive ductal carcinoma with axillary lymph node metastasis. She had a five year history of CD5 positive chronic lymphocytic leukaemia, which never required treatment. Immunoperoxidase stains for CD5, using the monoclonal antibody NCL-CD-54C7, showed that there was extensive infiltration of axillary lymph nodes with CD5 positive B lymphocytes. Strong staining for CD5 was also seen in the carcinoma cells within the breast and lymph node metastases. It has recently been suggested that there is a tumour suppresser locus in chronic lymphocytic leukaemia at 13q12.3 near or at the BRCA2 locus. Deletion of regions on chromosome 13q containing the BRCA2 and $\mathrm{RB} 1$ genes has also been reported in sporadic breast cancers. These observations suggest that there may be a link between these two diseases acting through chromosome 13, but amplification of several microsatellite repeat markers failed to show any loss of heterozygosity or repeat instability at either these or several other loci on chromosome 13. Examination of additional such cases is needed to perform a more comprehensive study of the significance of positive CD5 staining of breast carcinoma.

(f Clin Pathol 1998;51:862-864)
\end{abstract}

Keywords: breast carcinoma; chronic lymphocytic leukaemia; CD5; chromosome 13

Chronic lymphocytic leukaemia is characterised by the clonal expansion of neoplastic CD5+ B cells in the blood, bone marrow, lymph nodes, and spleen. ${ }^{1}$ It has recently been reported that 28 of 35 patients with chronic lymphocytic leukaemia showed deletion of the 13q12.3 locus, encompassing the BRCA2 gene, and homogeneous deletion of BRCA2 was detected in a significant proportion of cells in two thirds of these cases. ${ }^{1}$ Here we report the case of a patient with a history of untreated chronic lymphocytic leukaemia who developed breast carcinoma. The stroma of the tumour and the axillary lymph nodes were rich in CD5+ B lymphocytes, and the breast carcinoma cells were also positively stained for the CD5 antigen.

\section{Case report}

The patient was a 67 year old woman who developed a right breast lump. She had a history of chronic lymphocytic leukaemia which was diagnosed in March 1991. She had an absolute lymphocytosis of $10.7 \times 10^{9} /$ litre, most of the cells being small mature lymphocytes with some intermediate sized lymphocytes with abundant cytoplasm. Marker studies showed that the cells were CD5 and CD23 positive. There has been no evidence of progression of her leukaemia and she had never required treatment. At the time the patient presented with the breast lump her haemoglobin was $14.0 \mathrm{~g} / \mathrm{dl}$, white blood count $14.5 \times 10^{9} /$ litre, lymphocytic count $9.6 \times$ $10^{9} /$ litre, and platelet count $175 \times 10^{9} /$ litre. She appeared mildly immunosuppressed with a slightly low IgA concentration of $0.61 \mathrm{~g} /$ litre (normal 0.8-4.0). She had no family history of breast cancer and her BRCA2 status was not known.

The breast lump was initially treated by a wide local excision and axillary clearance, but as microscopic examination showed that the tumour was close to the resection margins, mastectomy was carried out later.

The excised tumour measured $4.0 \mathrm{~cm}$ in maximum dimension. Microscopically, it consisted of closely packed foci of grade 2 invasive ductal carcinoma with extensive central fibrosis and elastosis and scattered focal aggregates of lymphocytes. Foci of high grade, solid and comedo ductal carcinoma in situ were present in the vicinity of the invasive tumour. Widespread lymphatic invasion was present. Metastatic carcinoma was seen in three of $20 \mathrm{lymph}$ nodes dissected from the axilla. Parts of the metastatic tumour were much less differentiated than the primary breast tumour and included many tumour giant cells. Immunoperoxidase stains, using paraffin sections, showed extensive HCG positivity in the metastatic tumour cells as well as patchy HCG staining of some primary tumour cells.

For the demonstration of CD5 we used the avidin-biotin immunoperoxidase technique and the specific monoclonal antibody NCLCD5-4C7 (Novocastra) in a concentration of $1 / 100$, after subjecting the paraffin sections to a step of pressure cooker antigen retrieval. Most of the lymphocytes in the axillary lymph nodes and breast tumour stroma were CD5 positive, with only a small minority also being CD3 positive (a marker for $\mathrm{T}$ cells). Widespread cytoplasmic CD5 positivity was seen in the pri- 


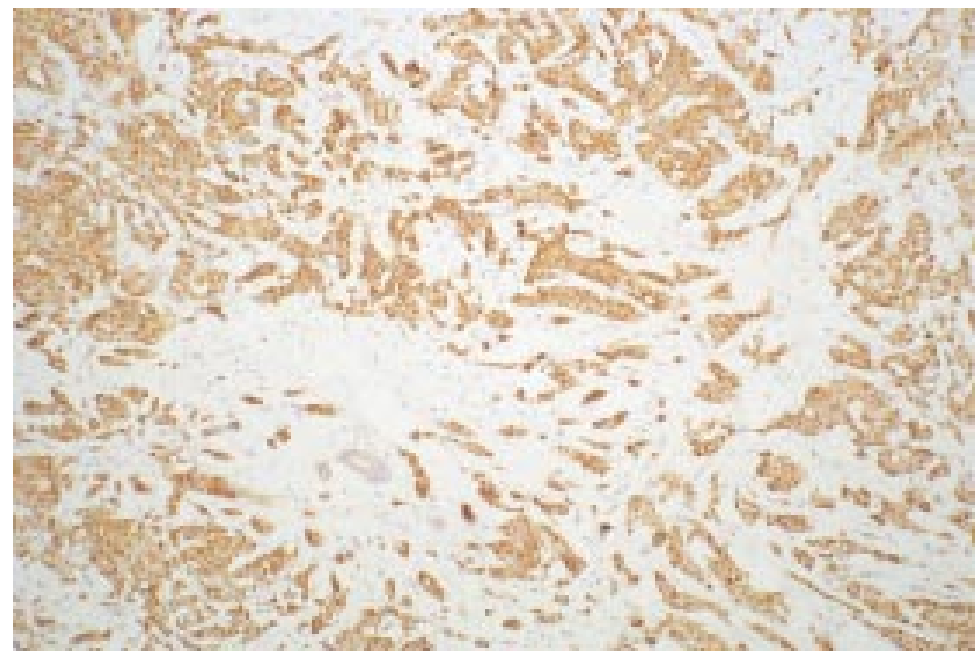

Figure 1 CD5 positive primary invasive ductal carcinoma. Positivity is seen as dark brown cytoplasmic staining. Avidin-biotin immunoperoxidase stain using the monoclonal antibody NCL-CD5-4C7 and light haematoxylin background staining.

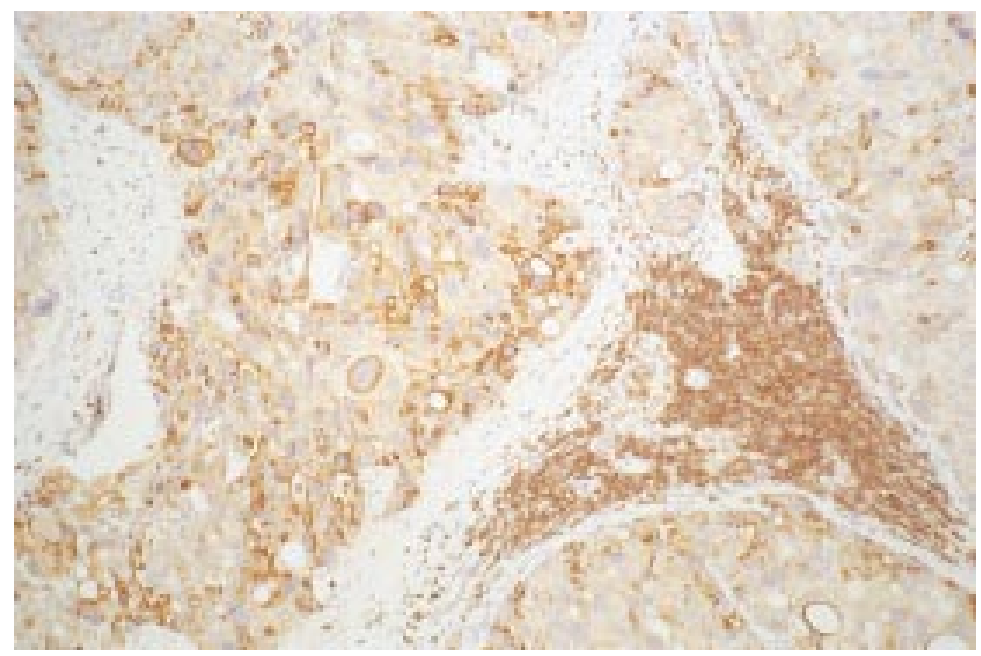

Figure 2 CD5 positive metastatic breast carcinoma in an axillary lymph node. Note abundant CD5 positive lymphocytes in the centre of the field. Avidin-biotin

immunoperoxidase stain using the monoclonal antibody NCL-CD5-4C7 as in fig 1.

mary (fig 1) and metastatic (fig 2) breast carcinoma cells. Further immunohistochemical studies showed that the tumour was strongly positive for oestrogen receptor ( $\mathrm{H}$ score $300 / 300$ ), progesterone receptor negative (0), and cerb-B2, p53, and $\mathrm{Rb}$ positive.

DNA was extracted, as described previously, ${ }^{2}$ from $7 \mu \mathrm{M}$ thick sections cut from three paraffin blocks, one containing tumour, the second normal breast tissue, and the third axillary lymph node tissue with no metastatic tumour. The DNA was further purified by passage through Cleanmix resin (Talent srl, Italy) and then $1 / 50$ th of each sample was amplified in a $25 \mu \mathrm{l}$ mix using conditions described elsewhere, ${ }^{3}$ except that the buffer contained $3 \mathrm{mM} \mathrm{MgCl}$ and the annealing temperature was $57^{\circ} \mathrm{C}$. The primers used to amplify dinucleotide repeats on chromosome 13 were D13S285 and 13q34; the primers used to amplify tetranucleotide repeats were D13S787; 13q11, D13S1493; 13pter, D13S894; 13q12.3, D13S325; 13q14.1, D13S317; 13q22, D13S796; and 13q32. PCR products were analysed on denaturing polyacrylamide gels containing $7 \mathrm{M}$ urea and visualised by silver staining. ${ }^{3}$ In all cases the same bands were seen for all three samples. There was no loss of heterozygosity or evidence of microsatellite instability at any of these sites.

\section{Discussion}

Although patients with chronic lymphocytic leukaemia are at increased risk of developing second malignant neoplasms, recent reports suggest that they may actually have a lower than average incidence of breast carcinoma, possibly because of the effect on ovarian function of chemotherapeutic agents used in the treatment of some cases. ${ }^{4}$ However, families with BRCA2 germ line mutation, breast cancer, and leukaemia have been reported, ${ }^{5}$ although no details about the type of leukaemia were provided in these cases. The recent suggestions about the existence of a tumour suppresser locus in chronic lymphocytic leukaemia at 13q12.3 near or at the BRCA2 gene, ${ }^{1}$ and the reported frequent presence in sporadic breast cancer of deleted regions on chromosome $13 \mathrm{q}$ containing BRCA2 or RB genes, ${ }^{7}$ provide a possible link between these two diseases through chromosome 13. Interestingly, allelic loss on chromosome $13 \mathrm{q}$ was reported in $39 \%$ of sporadic breast carcinomas of all types, and in $55 \%$ of the "solid tubular" type. ${ }^{7}$ We did not find evidence of loss in this particular patient, either at these sites or at a few other points along chromosome 13 , and immunostaining for $\mathrm{Rb}$ was positive in the carcinoma cells.

The CD5 molecule (T1, Leu 1) is a $67 \mathrm{kDa}$ transmembrane glycoprotein that displays features common to many growth factor receptors, including a high cysteine content in the extracellular domain and a large cytoplasmic region with a potential tyrosine phosphorylation site. ${ }^{8-10}$ It is normally present in almost all $\mathrm{T}$ lymphocytes and in a subset of B lymphocytes. ${ }^{10}$ To our knowledge, the presence of CD5 positivity in breast carcinoma has not been reported before. Our preliminary investigations, carried out on 17 consecutive cases, suggest that CD5 positivity-as demonstrated by NCL-CD5-4C7 antibody-is present in around $60 \%$ of breast carcinomas (10/17), but its distribution in positive cases varies widely, as well as its association with increased numbers of breast CD 5 positive lymphocytes (unpublished observations). The presence of intense CD5 positivity within the tumour cells in the current case, in association with increased numbers of CD5 positive B lymphocytes in the blood, axillary lymph nodes, and breast tissue, may provide a new morphological link between chronic lymphocytic leukaemia, breast carcinoma, and possibly unidentified specific chromosomal abnormalities in patients who develop, or are at risk of developing, the two diseases simultaneously or consecutively.

1 Garcia-Marco JA, Caldas C, Price CM, et al. Frequent somatic deletion of the 13q12.3 locus encompassing BBCA2 in chronic lymphocytic leukemia. Blood

2 Luqmani YA, Shousha S. Presence of Epstein-Barr virus in

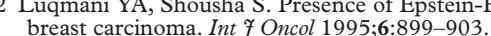

3 Luqmani YA, Mathew M, Temmim L, et al. Separation of microsatellite fragments on small precast polyacrylamide 
gels and visualisation by silver staining. Anal Biochem 1997;253:130-2

4 Travis LB, Curtis RE, Hankey BF, et al. Second cancers in patients with chronic lymphocytic leukemia. $\mathcal{F}$ Natl Cancer

5 Goldgar DE, Neuhausen SL, Steele L, et al. A 45-year follow-up of kindred 107 and the search for BRCA2 Monogr Natl Cancer Inst 1995;17:15-19.

6 Berman DB, Costalas J, Schultz DC, et al. A common mutation in BRCA2 that predisposes to a variety of cancers is found in both Jewish Ashkenazi and non-Jewish individuals. Cancer Res 1996;56:3409-14.

7 Tsukamoto K, Ito N, Yoshimoto M, et al. Two distinct commonly deleted regions on chromosome $13 \mathrm{q}$ suggest involvement of BRCA2 and retinoblastoma genes in sporadic breast carcinomas. Cancer 1996;78:1929-34.

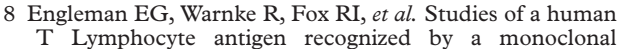
antibody. Proc Natl Acad Sci USA 1981;78:1791-5.

9 Jones NH, Clabby ML, Dialynas DP, et al. Isolation of complementary DNA clones encoding the human lymphocyte glycoprotein T1/Leu-1. Nature 1986;323:346-9.

10 Huang H-J S, Jones NH, Strominger JL, et al. Molecular cloning of Ly-1, a membrane glycoprotein of mouse $\mathrm{T}$ lymphocytes and a subset of B cells: molecular homology to its human counterpart Leu-1/T1 (CD5). Proc Natl Acad Sci USA 1987;84:204-8. 